\title{
ON THE ABELIAN FIVEFOLDS ATTACHED TO CUBIC SURFACES
}

\author{
JefFrey D. ACHTER
}

\begin{abstract}
To a family of smooth projective cubic surfaces one can canonically associate a family of abelian fivefolds. In characteristic zero, we calculate the Hodge groups of the abelian varieties which arise in this way. In arbitrary characteristic, we calculate the monodromy group of the universal family of abelian varieties, and thus show that the Galois group of the 27 lines on a general cubic surface in positive characteristic is as large as possible.
\end{abstract}

\section{Introduction}

The moduli space of smooth complex cubic surfaces is open in an arithmetic quotient of the complex 4-ball $[3,11]$. This statement is the complex specialization of a homeomorphism $\tau$ of stacks over $\mathbb{Z}\left[\zeta_{3}, 1 / 6\right]$ between $\mathcal{S}_{\text {st }}$, the moduli space of stable cubic surfaces, and $\mathcal{M}$, the moduli space of principally polarized abelian fivefolds equipped with an action by $\mathbb{Z}\left[\zeta_{3}\right]$ of signature $(4,1)[1]$. In particular, to a cubic surface $Y$ one may canonically associate an abelian fivefold $X=Q(Y)$; it is natural to try and characterize the abelian varieties which arise in this way.

Different strategies have the potential to yield different information. For example, it is possible [1, Corollary 5.9] to reconstruct $Y$ from the theta divisor of the polarized abelian variety $X$. However, this geometric method does not obviously allow one to understand, say, the endomorphism ring $\operatorname{End}(X)$. Alternatively, at least over the complex numbers, one can (as Carlson and Toledo do [7]) explicitly evaluate the necessary period integrals. This approach has the virtue of explicitly relating symmetries of $Y$ to endomorphisms of $X$, but seems unlikely to yield an explicit classification of all possible abelian varieties.

The main theme of the present paper is that, via $\tau$, techniques from the theory of Shimura varieties (and their compactifications) can be brought to bear on the moduli space of cubic surfaces.

In Section 3, we completely classify the Hodge groups of complex abelian fivefolds of the form $Q(Y)$. Proposition 3.6 describes the endomorphism algebras, and thus the Hodge groups, which could conceivably arise in this way, while Proposition 3.12 guarantees that such behavior really does occur.

In Section 4, we calculate the monodromy group of the universal abelian variety when pulled back to $\mathcal{S}$. In fact, the Torelli map $\tau$ passes through the moduli stack $\mathcal{T}$ of cubic threefolds, and we also calculate the monodromy group of the universal abelian variety over $\mathcal{T}$ (Theorem 4.3). As a consequence, we are able to show

Received by the editors October 4, 2012.

1991 Mathematics Subject Classification. Primary 14J10; Secondary 11G18, 14D05, 14 K30. 
(Corollary 4.7) that there are cubic surfaces $Y$ defined over $\mathbb{Q}$ with endomorphism ring exactly equal to $\mathbb{Z}\left[\zeta_{3}\right]$. The paper concludes by using the theory of arithmetic toroidal compactifications of Shimura varieties of PEL type in order to compute the Galois group of the 27 lines on a sufficiently general cubic surface in characteristic at least five (Proposition 4.8).

The classification in Section 3 and the monodromy calculation in Section 4 require, respectively, a method for constructing principally polarized abelian varieties with specified endomorphism ring and information about unitary Shimura varieties. These are developed in a broader context than that required for understanding cubic surfaces. This generality imposes no extra burden on the proof, and these results may be of independent interest.

Given a polarized abelian variety $(X, \lambda)$ and an order $\mathcal{O} \subset \operatorname{End}(X)$, by adjusting $(X, \lambda)$ in its isogeny class one can either find a principal polarization, or expand $\mathcal{O}$ to a maximal order; but achieving both simultaneously seems delicate. Section 3.4, especially Lemma 3.11, gives conditions under which one can find a principally polarized abelian variety with specified commutative endomorphism algebra and an action by the ring of integers of a quadratic imaginary field of specified signature.

The mod- $\ell$ monodromy calculation for $\mathcal{S}$ comes down to a question of the irreducible components of $\mathcal{M}^{\ell}$, the moduli space of $\mathbb{Z}\left[\zeta_{3}\right]$-abelian varieties equipped with a principal level $\ell$ structure. This is worked out in detail in Lemma 4.5 for an arbitrary PEL Shimura variety attached to $\mathbb{Z}\left[\zeta_{3}\right]$; the proof relies both on class field theory and on arithmetic compactifications.

\section{Background and notation}

The following notation will be in force throughout the paper, except that: in Section 3.4, $E$ denotes an arbitrary quadratic imaginary field, and not necessarily $\mathbb{Q}\left(\zeta_{3}\right)$; and in Section $4.2, \mathcal{M}$ denotes the moduli space of abelian varieties with $\mathbb{Z}\left[\zeta_{3}\right]$-action of arbitrary, fixed signature $(r, s)$, and not necessarily signature $(4,1)$.

2.1. Moduli spaces. Let $\mathcal{O}_{E}=\mathbb{Z}\left[\zeta_{3}\right]$ be the ring of Eisenstein integers, with fraction field $E=\mathbb{Q}\left(\zeta_{3}\right)$.

The present work explores some consequences of the existence of nontrivial maps between moduli spaces of cubic surfaces, cubic threefolds, and abelian fivefolds.

Let $\mathcal{S}$ be the moduli stack of smooth cubic surfaces. It is the quotient of $\widetilde{\mathcal{S}}$, the moduli scheme of smooth cubic forms in four variables, and is partially compactified by $\mathcal{S}_{\text {st }}$, the moduli stack of stable cubic surfaces. Similarly, let $\mathcal{T}$ be the moduli stack of smooth cubic threefolds, and let $\widetilde{\mathcal{T}}$ be the moduli scheme of smooth cubic forms in five variables.

Finally, let $\mathcal{A}_{5}$ be the moduli stack of principally polarized abelian fivefolds, and let $\mathcal{M}$ be the moduli stack of principally polarized abelian fivefolds equipped with an action by $\mathbb{Z}\left[\zeta_{3}\right]$ of signature $(4,1)$. 
The main work of [1] is to define and characterize the morphisms $\widetilde{\varpi}$ (over $\mathbb{Z}[1 / 2]$ ) and $\tau$ (over $\mathbb{Z}\left[\zeta_{3}, 1 / 6\right]$ ) indicated below:

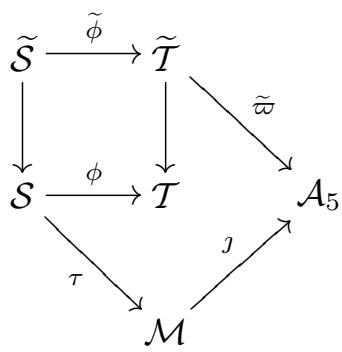

In this diagram, $\phi$ is the functor which, to a cubic surface $Y \subset \mathbb{P}^{3}$, associates the cubic threefold $Z \subset \mathbb{P}^{4}$ which is the cyclic triple cover of $\mathbb{P}^{3}$ ramified exactly along $Y$. The morphism $\widetilde{\varpi}$ comes from an arithmetic Prym construction; over the complex numbers, it coincides with the intermediate Jacobian functor. (It seems plausible that $\widetilde{\varpi}$ factors through $\mathcal{T}$, but the author has not checked this.)

Let $\mathcal{D} \subset \mathcal{M}$ be the substack parametrizing $\mathbb{Z}\left[\zeta_{3}\right]$-abelian schemes which are isomorphic to the product of an elliptic curve and an abelian fourfold. It is a horizontal divisor; let $\mathcal{N}=\mathcal{M}-\mathcal{D}$. The main result of [1] is:

Theorem 2.1 ([1, Theorem 5.7]). There is an isomorphism of stacks $\tau: \mathcal{S} \rightarrow \mathcal{N}$ over $\mathbb{Z}\left[\zeta_{3}, 1 / 6\right]$ which extends to a homeomorphism of stacks $\mathcal{S}_{\text {st }} \rightarrow \mathcal{M}$.

(On the boundary, the existence of nodal cubic surfaces without nontrivial automorphisms means that, even over $\mathbb{C},\left(\mathcal{S}_{\text {st }} \backslash \mathcal{S}\right)_{\mathbb{C}} \rightarrow \mathcal{D}_{\mathbb{C}}$ is not an isomorphism of stacks; see $[3,3.18]$ for details.)

The universal abelian fivefold, cubic surface and cubic threefold will be respectively denoted by $f: \mathcal{X} \rightarrow \mathcal{A}_{5}, g: \mathcal{Y} \rightarrow \mathcal{S}$ and $h: \mathcal{Z} \rightarrow \mathcal{T}$; we will abuse notation and use the same letters for the universal objects over $\widetilde{\mathcal{S}}$ and $\widetilde{\mathcal{T}}$. The middle cohomology of $\mathcal{Z}$ is a Tate twist of the first cohomology of $\widetilde{\varpi}^{*} \mathcal{X}$; see $[1$, Proposition 3.6] for a precise statement.

As a shorthand for the Prym functor, for $t \in \widetilde{\mathcal{T}}$ let $P\left(\mathcal{Z}_{t}\right)=\mathcal{X}_{\widetilde{\varpi}(t)}$. Thus, if $Z$ is a cubic threefold, $P(Z)$ is the principally polarized abelian fivefold attached to $Z$ by $\widetilde{\varpi}$. Similarly, let $F(Y)$ be the cubic threefold which is the cyclic triple cover of $\mathbb{P}^{3}$ ramified along $Y ; F\left(\mathcal{Y}_{s}\right)=\mathcal{Z}_{\phi(s)}$. Finally, let $Q(Y)=P(F(Y))$.

If $X$ is an abelian variety, denote its endomorphism algebra by $\operatorname{End}(X)_{\mathbb{Q}}=$ $\operatorname{End}(X) \otimes \mathbb{Q}$.

2.2. Unitary groups. Let $V=\mathcal{O}_{E}^{\oplus 5}$, and equip it with the Hermitian pairing

$$
\langle\langle x, y\rangle\rangle=\sum_{1 \leq j \leq 4} x_{j} \bar{y}_{j}-x_{5} \bar{y}_{5} .
$$

Then $\langle x, y\rangle=\operatorname{tr}(\langle\langle x, y\rangle\rangle / \sqrt{-3})$ is a symplectic, $\mathbb{Z}$-linear pairing on the underlying $\mathbb{Z}$-module of $V$, and $\langle a x, y\rangle=\langle x, \bar{a} y\rangle$ for $a \in \mathcal{O}_{E}$. Define various unitary group 
schemes over $\mathbb{Z}$ as follows:

$$
\begin{aligned}
\mathrm{GU} & =\mathrm{GU}(V,\langle\langle\cdot, \cdot\rangle\rangle) \\
\mathrm{U} & =\mathrm{U}(V,\langle\langle\cdot, \cdot\rangle\rangle) \\
\mathrm{SU} & =\mathrm{SU}(V,\langle\langle\cdot, \cdot\rangle\rangle)
\end{aligned}
$$

and the tori

$$
\begin{aligned}
\mathrm{T} & =\mathbf{R}_{\mathcal{O}_{E}, \mathbb{Z}} \mathbb{G}_{m, E}, \\
\mathrm{~T}_{1} & =\mathbf{R}_{\mathcal{O}_{E}, \mathbb{Z}}\left(\operatorname{ker} \mathcal{N}_{\mathcal{O}_{E} / \mathbb{Z}}\right),
\end{aligned}
$$

where $\mathbf{R}_{\mathcal{O}_{E} / \mathbb{Z}}$ is the restriction of scalars functor. Then $\mathrm{SU}$ is the derived group of GU and of $\mathrm{U}$, and there are the following sequences of group schemes, exact as sheaves on $(\operatorname{Spec} \mathbb{Z}[1 / 6])^{\text {et }}$ :

$$
\begin{aligned}
& 1 \longrightarrow \mathrm{SU} \longrightarrow \mathrm{GU} \stackrel{\nu}{\longrightarrow} \mathrm{T} \longrightarrow 1 \\
& 1 \longrightarrow \mathrm{SU} \longrightarrow \mathrm{U} \stackrel{\nu}{\longrightarrow} \mathrm{T}_{1} \longrightarrow 1
\end{aligned}
$$

There is a natural inclusion of group schemes $\mathbf{R}_{\mathcal{O}_{E} / \mathbb{Z}} \boldsymbol{\mu}_{6} \hookrightarrow \mathrm{T}_{1}$. Let

$$
\mathrm{U}^{*}=\mathrm{U} \times_{\mathrm{T}_{1}} \boldsymbol{\mu}_{6},
$$

an extension of $\boldsymbol{\mu}_{6}$ by SU.

For each natural number $N, \mathrm{SU}(\mathbb{Z}) \rightarrow \mathrm{SU}(\mathbb{Z} / N)$ is surjective (Lemma 4.4). Since $\mathrm{T}_{1}(\mathbb{Z})=\mathbf{R}_{\mathcal{O}_{E} / \mathbb{Z}} \boldsymbol{\mu}_{6}(\mathbb{Z})$ is the group of sixth roots of unity (and not larger), $\mathrm{U}^{*}(\mathbb{Z} / N)$ is the image of the reduction $\operatorname{map} \mathrm{U}(\mathbb{Z}) \rightarrow \mathrm{U}(\mathbb{Z} / N)$.

\section{Mumford-Tate groups and endomorphism rings}

Carlson and Toledo have raised the question of classifying the Mumford-Tate groups of abelian varieties associated to complex cubic surfaces. In this section, we avail ourselves of known results on endomorphisms of abelian varieties in order to classify the algebras $\operatorname{End}(Q(Y))_{\mathbb{Q}}$ as $Y$ ranges over all smooth complex cubic surfaces. Briefly, we find that each abelian variety has at most one simple factor of dimension greater than one; this yields a coarse classification by "shape", i.e., by the dimension of the largest simple factor and the dimension of its endomorphism algebra. After an apparent digression on the problem of constructing principally polarized abelian varieties with large endomorphism ring (as opposed to endomorphism algebra), we are able to show that every possible shape occurs in $\mathcal{S}$.

Carlson and Toledo have independently investigated the Mumford-Tate groups of the abelian varieties which arise here, and the reader is invited to consult [7] for a complementary perspective which emphasizes period integrals.

3.1. Topological monodromy. Consider the spaces of complex cubic surfaces and threefolds. By pulling back the middle cohomology of the universal family of cubic threefolds, one obtains a local system $\widetilde{\phi}^{*} R^{3} h_{*} \mathbb{Z}$ on $\widetilde{S}_{\mathbb{C}}$ of polarized $\mathbb{Z}\left[\zeta_{3}\right]$-modules of rank 5 equipped with a Hermitian pairing. Fix a base point $s \in \widetilde{S}(\mathbb{C})$; the local system $\widetilde{\phi}^{*} R^{3} h_{*} \mathbb{Z}$ is determined by a representation $\pi_{1}^{\top}\left(\widetilde{S}_{\mathbb{C}}, s\right) \rightarrow \operatorname{Aut}\left(H^{3}\left(\mathcal{Z}_{\phi(s)}, \mathbb{Z}\right)\right)$ of the 
topological fundamental group of $\widetilde{S}_{\mathbb{C}}$, whose image is simply called the (topological) monodromy group of $\mathcal{Y}_{\mathbb{C}} \rightarrow \widetilde{\mathcal{S}}_{\mathbb{C}}$.

Proposition 3.1 ([3, Theorem 2.14]). The monodromy group of the universal family of cubic surfaces is a subgroup $\mathrm{U}^{+}(\mathbb{Z}) \subset \mathrm{U}(\mathbb{Z})$ of index two.

In fact, $[3]$ gives a beautifully explicit characterization of $\mathrm{U}^{+}(\mathbb{Z})$. For present purposes, it suffices to note that since $\mathrm{SU}(\mathbb{Z})$ has no subgroup of index two, $U^{+}(\mathbb{Z})$ must contain $\mathrm{SU}(\mathbb{Z})$ and is therefore an extension of $\boldsymbol{\mu}_{3}$ by $\mathrm{SU}(\mathbb{Z})$. This will be used below (Section 3.2) in describing Mumford-Tate groups of intermediate Jacobians of cubic threefolds attached to cubic surfaces.

The comparison theorem between Betti and étale cohomology lets one conclude that the connected component of the geometric $\overline{\mathbb{Q}}_{\ell}$-monodromy group of $\mathcal{Y} \rightarrow \widetilde{\mathcal{S}}$ is $\mathrm{SU}_{\overline{\mathbb{Q}}_{\ell}}$. However, a direct calculation (Theorem 4.3) below yields more detailed information (see Remark 4.2) about the monodromy group of the étale cohomology.

3.2. Hodge groups and Mumford-Tate groups. Let $X / \mathbb{C}$ be a complex abelian variety. Then $W:=H^{1}(X(\mathbb{C}), \mathbb{Q})$ is a polarizable Hodge structure. If the Hodge structure on $W$ is realized as a map of $\mathbb{R}$-groups $h: \mathbf{R}_{\mathbb{C} / \mathbb{R}} \mathbb{G}_{m} \rightarrow \operatorname{Aut}\left(W \otimes_{\mathbb{Q}} \mathbb{R}\right)$, then the Mumford-Tate group $\mathrm{MT}(W)$ is the smallest algebraic subgroup $M / \mathbb{Q}$ such that $h$ factors through $M_{\mathbb{R}}$. The Hodge structure is called special if $\mathrm{MT}(W)$ is a torus.

In a similar sense, the Hodge group $\mathrm{Hg}(W)$ of $W$ is the algebraic hull of the image of the restriction of $h$ to the unit circle in $\mathbb{C}$. From this description, it is clear that $\operatorname{MT}(W)$ is an extension of $\mathbb{G}_{m}$ by $\mathrm{Hg}(W)$. Let $\mathfrak{h} \mathfrak{g}(W)$ be the Lie algebra of $\mathrm{Hg}(W)$. Finally, let $\operatorname{MT}(X), \operatorname{Hg}(X)$ and $\mathfrak{h} \mathfrak{g}(X)$ denote the corresponding objects for $W$.

Now consider $\mathbb{W}=\widetilde{\phi}^{*} R^{3} h_{*} \mathbb{Q}$ as a polarized variation of Hodge structure on $\widetilde{\mathcal{S}}_{\mathbb{C}}$. There is a generic Mumford-Tate group $\mathrm{MT}^{\text {gen }}(\mathbb{W})$ associated to $\mathbb{W}$. It is characterized by the fact that for each $s \in \widetilde{\mathcal{S}}(\mathbb{C}), \mathrm{MT}\left(\mathbb{W}_{s}\right) \subseteq \mathrm{MT}^{\text {gen }}(\mathbb{W})$; and for $s$ in the complement of a certain countable union of proper (a priori analytic, but actually algebraic) subvarieties of $\widetilde{\mathcal{S}}$, there is equality $\operatorname{MT}\left(\mathbb{W}_{s}\right) \cong \mathrm{MT}^{\text {gen }}(\mathbb{W})$.

Lemma 3.2. The generic Mumford-Tate group of $\mathbb{W}$ is $\mathrm{MT}^{\mathrm{gen}}(\mathbb{W})=\mathrm{GU}_{\mathbb{Q}}$.

Proof. Since $\mathcal{M}$ is a Shimura variety, special points are analytically dense in $\mathcal{M}_{\mathbb{C}}$, and thus in $\widetilde{\mathcal{S}}_{\mathbb{C}}$. Consequently [4, Proposition 2], the derived subgroup of $\mathrm{MT}^{\text {gen }}(\mathbb{W})$ is equal to the connected component of the (Zariski closure of) the topological monodromy group. The result now follows from Proposition 3.1.

In order to classify all groups which arise as Mumford-Tate groups of abelian varieties associated to cubic surfaces, in principle one could work out all sub-Shimura data of the (connected) Shimura datum $\left(U, B^{4}\right)$. Instead, we proceed as follows. The Mumford-Tate group of an abelian variety is invariant under isogeny of the abelian variety. The Hecke orbit of each $s \in \mathcal{M}(\mathbb{C})$ is analytically dense. In particular, each point in $\mathcal{D}(\mathbb{C})$, the complement of the Torelli map $\mathcal{S}(\mathbb{C}) \rightarrow \mathcal{M}(\mathbb{C})$, is isogenous to one in $\mathcal{N}(\mathbb{C})=(\mathcal{M} \backslash \mathcal{D})(\mathbb{C})$, and thus every Mumford-Tate group which arises in $\mathcal{M}$ already arises in the variation of Hodge structure $\mathbb{W} \rightarrow \widetilde{\mathcal{S}}_{\mathbb{C}}$. It turns out that, for a complex abelian fivefold with action by $\mathbb{Z}\left[\zeta_{3}\right]$, the endomorphism algebra completely determines the Mumford-Tate group. Consequently, we proceed by classifying endomorphism algebras and then listing the associated Mumford-Tate (actually, Hodge) groups. 
3.3. Mumford-Tate groups and endomorphism algebras. In this section we work up to isogeny, and in particular "simple" means "simple up to isogeny".

As a preview, note that if one is willing to restrict attention to abelian fivefolds with $\mathbb{Z}\left[\zeta_{3}\right]$-action which are simple up to isogeny, then the calculation is much shorter. Indeed, suppose $X=(X, \iota, \lambda) \in \mathcal{M}(\mathbb{C})$ is simple. Then, using the fact that $\operatorname{dim}(X)=$ 5 is prime, one quickly sees that $\operatorname{End}(X)_{\mathbb{Q}}$ is either $\mathbb{Q}\left(\zeta_{3}\right)$ or a CM field $K$ such that $[K: \mathbb{Q}]=10$. In the former case, the abelian variety has Mumford-Tate group equal to $\mathrm{MT}^{\mathrm{gen}}(\mathbb{W}) \cong \mathrm{GU}_{\mathbb{Q}}$. In the latter case, $K$ is necessarily the compositum of $\mathbb{Q}\left(\zeta_{3}\right)$ and $K_{0}$, the maximal totally real subfield of $K$, and the Mumford-Tate group of the abelian variety is a torus. The existence of the two kinds of points over number fields is explored in Corollary 4.7 and [7, Section 4], respectively.

Let $X_{0} / \mathbb{C}$ be the elliptic curve with action by $\mathbb{Z}\left[\zeta_{3}\right]$ of signature $(1,0)$.

Suppose $B$ is an arbitrary abelian variety. Then $\operatorname{End}(B \times B)$ is canonically isomorphic to $\mathrm{Mat}_{2}(\operatorname{End}(B))$. Via the embeddings

$$
\mathbb{Z}\left[\zeta_{3}\right] \longrightarrow \operatorname{End}_{\mathbb{Z}}\left(\mathbb{Z}\left[\zeta_{3}\right]\right) \stackrel{\sim}{\longrightarrow} \operatorname{Mat}_{2}(\mathbb{Z}) \longleftrightarrow \operatorname{Mat}_{2}(\operatorname{End}(B)) \stackrel{\sim}{\longrightarrow} \operatorname{End}(B \times B),
$$

$B \times B$ admits an action by $\mathbb{Z}\left[\zeta_{3}\right]$ of signature $(\operatorname{dim} B, \operatorname{dim} B)$.

Lemma 3.3. If $X$ is a complex abelian fivefold with action by $\mathbb{Z}\left[\zeta_{3}\right]$ of signature $(4,1)$, then $X$ is isogenous, as an abelian variety with $\mathbb{Z}\left[\zeta_{3}\right]$-action, to an abelian variety of exactly one of the following forms:

(a) $X_{0}^{3} \times X_{1}^{2}$, where $X_{1}$ is an elliptic curve which is not isogenous to $X_{0}$.

(b) $X_{0}^{(5-n)} \times X_{n}$, where $1 \leq n \leq 5$ and $X_{n}$ is a simple abelian variety of dimension $n$ with an action by $\mathbb{Z}\left[\zeta_{3}\right]$ of signature $(n-1,1)$.

Remark 3.4. The case $n=5$ was already described at the end of Section 3.2.

Proof. Suppose $A$ is an abelian variety with an action by $\mathbb{Z}\left[\zeta_{3}\right]$. If $A$ is simple as a $\mathbb{Z}\left[\zeta_{3}\right]$-abelian variety, then either it is actually simple, or it decomposes as $A \sim B \times B$. In the latter case, $B$ does not admit an $\mathbb{Z}\left[\zeta_{3}\right]$-action, and the signature of the $\mathbb{Z}\left[\zeta_{3}\right]$ action on $A$ is $(\operatorname{dim} B, \operatorname{dim} B)$. Moreover, if $X_{1}$ and $X_{2}$ are abelian varieties with $\mathbb{Z}\left[\zeta_{3}\right]$ actions of respective signatures $\left(r_{1}, s_{1}\right)$ and $\left(r_{2}, s_{2}\right)$, then the signature of $X_{1} \times X_{2}$ is $\left(r_{1}+r_{2}, s_{1}+s_{2}\right)$. Consequently, if $X$ is a $\mathbb{Z}\left[\zeta_{3}\right]$-abelian variety of signature $(4,1)$, then it admits at most one factor which is simple as $\mathbb{Z}\left[\zeta_{3}\right]$-abelian variety but not as an abelian variety; and this factor must be the self-product of an elliptic curve. This is case (a). Now suppose that $X$ has no such factor. A simple complex abelian variety $B$ with $\mathbb{Z}\left[\zeta_{3}\right]$ action of signature $(r, 0)$ necessarily has $r=\operatorname{dim} B=1[28$, Proposition 14]. Consequently, $X$ has at most one simple factor of dimension greater than one. The classification asserted in the lemma now follows.

We now take up the task of describing the possibilities for the endomorphism algebra of a simple abelian variety with $\mathbb{Z}\left[\zeta_{3}\right]$-action.

If $K$ is a CM field, with maximal totally real subfield $K_{0}$, let $\mathrm{U}_{K}$ be the norm-one torus

$$
\mathrm{U}_{K}=\operatorname{ker}\left(\mathbf{R}_{K / \mathbb{Q}} \mathbb{G}_{m, K} \stackrel{\mathcal{N}_{K / K_{0}}}{\rightarrow} \mathbf{R}_{K_{0} / \mathbb{Q}} \mathbb{G}_{m, K_{0}}\right) .
$$

Let $(X, \lambda)$ be a simple polarized abelian variety, and let $W=H^{1}(X, \mathbb{Q})$. The polarization $\lambda$ induces an involution $(\dagger)$, the Rosati involution, on $\operatorname{End}(X)_{\mathbb{Q}}$ and on $\operatorname{End}(W)$. Suppose that $K$ is contained in the center $C(X)$ of $\operatorname{End}(X)_{\mathbb{Q}}$, and further assume 
that $(\dagger)$ restricts to complex conjugation on $K$. (Given $X$ and $K$, such a polarization always exists [19, Lemma 9.2].) Define the group

$$
\mathrm{U}_{K}(X, \lambda)=\left\{\alpha \in \operatorname{End}_{K}(W): \alpha \alpha^{(\dagger)}=1\right\}
$$

with Lie algebra

$$
\mathfrak{u}_{K}(X, \lambda)=\left\{\alpha \in \operatorname{End}_{K}(W): \forall v, w \in W: \lambda(\alpha(v), w)+\lambda(v, \alpha(w))=0\right\} .
$$

In the absence of a subscript, $\mathrm{U}(X, \lambda)$ will denote $\mathrm{U}_{C(X)}(X, \lambda)$. There is an a priori inclusion $\mathfrak{h} \mathfrak{g}(X) \subseteq \mathfrak{u}(X, \lambda)$.

Further suppose that $C(X)$ contains $E$. Then let $\operatorname{det}_{C(X) / E}$ denote the composition

$$
\mathrm{U}(X, \lambda) \stackrel{\operatorname{det}_{C(X)}}{\longrightarrow} \mathrm{U}_{C(X)} \stackrel{\mathcal{N}_{C(X) / E}}{\longrightarrow} \mathrm{U}_{E}
$$

(Note that we allow the possibility $C(X)=E$, in which case the second map is an isomorphism; or $2 \operatorname{dim} X=[C(X): \mathbb{Q}]$, in which case the first map may be an isomorphism.)

Lemma 3.5. Let $X / \mathbb{C}$ be a simple abelian variety of dimension $g \leq 5$ with an action by $\mathbb{Z}\left[\zeta_{3}\right]$ of signature $(g-1,1)$. If $g=2$, then $\operatorname{End}(X)_{\mathbb{Q}}$ is an indefinite quaternion algebra. If $g \neq 2$, then $\operatorname{End}(X)_{\mathbb{Q}}$ is the compositum of $\mathbb{Q}\left(\zeta_{3}\right)$ and a totally real field of dimension dividing $g$.

Proof. This can be deduced from the usual classification of endomorphism algebras of abelian varieties (see [25, Section 2]) and the following argument, which is adapted from $[24,2.9]$ and shows that $\operatorname{End}(X)_{\mathbb{Q}}$ is not a quaternion algebra if $g=4$. Let $(Y, \lambda) / \mathbb{C}$ be a simple polarized abelian variety with $\operatorname{End}(Y)_{\mathbb{Q}} \cong D$, a quaternion algebra over $\mathbb{Q}$; then $\operatorname{dim} Y=2 d$ is even. Now suppose that $\mathbb{Q}\left(\zeta_{3}\right)$ is a subalgebra of $D$ stable under the Rosati involution. We will show that the signature of the action of $\mathbb{Q}\left(\zeta_{3}\right)$ on $Y$ is $(d, d)$.

As noted above, there is an a priori inclusion $\mathfrak{h} \mathfrak{g}(Y) \subseteq \mathfrak{u}_{E}(Y, \lambda)$. Moreover, since the center of $D$ is totally real, $\mathfrak{h} \mathfrak{g}(Y)$ is semisimple [29, Lemma 1.4] and we have $\mathfrak{h} \mathfrak{g}(Y) \subseteq \mathfrak{s u}_{E}(Y, \lambda)$. Consequently, $Y$ has signature $(d, d)$ [24, Lemma 2.8].

We come to the main result of this section.

Proposition 3.6. If $X$ is a complex abelian fivefold with action by $\mathbb{Z}\left[\zeta_{3}\right]$ of signature $(4,1)$, then either:

(a) $X$ is isogenous to $X_{0}^{3} \times X_{1}^{2}$, where $X_{1}$ is an elliptic curve which is not isogenous to $X_{0}$, and $\mathrm{Hg}(X) \cong \mathrm{U}_{E} \times \mathrm{Hg}\left(X_{1}\right)$; or

(b) $X$ is isogenous to $X_{0}^{3} \times X_{2}$, where $X_{2}$ is a simple abelian surface. Then $D:=$ $\operatorname{End}(X)_{\mathbb{Q}}$ is an indefinite quaternion algebra,

$$
\operatorname{Hg}\left(X_{2}\right) \cong\left\{x \in\left(D^{\text {opp }}\right)^{\times}: x x^{(\dagger)}=1\right\}
$$

and

$$
\mathrm{Hg}(X) \cong \mathrm{U}_{E} \times \mathrm{Hg}\left(X_{2}\right)
$$


or

(c) $X$ is isogenous to $X_{0}^{(5-n)} \times X_{n}$, where $n \in\{1,3,4,5\}$ and $X_{n}$ is a simple abelian variety of dimension $n$ with an action by $\mathbb{Z}\left[\zeta_{3}\right]$ of signature $(n-1,1)$. Then $\operatorname{End}\left(X_{n}\right)_{\mathbb{Q}}$ is a CM field which is the compositum of $E$ and a totally real field. Let $\lambda_{n}$ be a polarization on $X_{n}$ for which the associated Rosati involution induces complex conjugation on $\operatorname{End}\left(X_{n}\right)_{\mathbb{Q}}$. Then

$$
\operatorname{Hg}\left(X_{n}\right) \cong \mathrm{U}\left(X_{n}, \lambda_{n}\right)
$$

(i) If $n=1$, then

$$
\mathrm{Hg}(X) \cong \mathrm{U}_{E}
$$

(ii) If $n=3$, then

$$
\operatorname{Hg}(X) \cong\left\{(u, v) \in \mathrm{U}_{E} \times \mathrm{U}\left(X_{n}, \lambda_{n}\right): u \cdot \operatorname{det}_{C(X) / E}(v)=1\right\} .
$$

(iii) If $n=4$, then

$$
\mathrm{Hg}(X) \cong\left\{(u, v) \in \mathrm{U}_{E} \times \mathrm{U}\left(X_{n}, \lambda_{n}\right): u^{2} \cdot \operatorname{det}_{C(X) / E}(v)=1\right\} .
$$

(iv) If $n=5$, then $X=X_{n}$ and thus

$$
\mathrm{Hg}(X) \cong \mathrm{U}(X, \lambda) \text {. }
$$

Remark 3.7. In part (c), a suitable choice of polarization $\lambda_{n}$ always exists [19, Lemma 9.2]. By definition, the abstract isomorphism class of $\operatorname{Hg}\left(X_{n}\right)$ is independent of the choice of polarization.

Proof. The Hodge group of an elliptic curve is either $\mathrm{SL}_{2}$ or a norm-one torus $\mathrm{U}_{K}$ for some quadratic imaginary field $K$. In particular, $\operatorname{Hg}\left(X_{0}\right) \cong \mathrm{U}_{E}$.

Suppose $Y_{1}, \ldots, Y_{m}$ are abelian varieties with $\operatorname{Hom}\left(Y_{i}, Y_{j}\right)=\{0\}$, and $e_{1}, \ldots, e_{m}$ are natural numbers. Then $\operatorname{Hg}\left(Y_{1}^{e_{1}} \times \cdots \times Y_{m}^{e_{m}}\right) \cong \operatorname{Hg}\left(Y_{1} \times \cdots \times Y_{m}\right)$. Recall the coarse classification established in Lemma 3.3. In case (a) of that lemma, we see that $\operatorname{Hg}(X) \cong \operatorname{Hg}\left(X_{0} \times X_{1}\right)$. Moreover, since $\operatorname{Hom}\left(X_{0}, X_{1}\right)=\{0\}, \operatorname{Hg}\left(X_{0} \times X_{1}\right) \cong$ $\operatorname{Hg}\left(X_{0}\right) \times \operatorname{Hg}\left(X_{1}\right)$.

Now suppose that $X$ falls in case (b) of Lemma 3.3. The assertions about $\operatorname{End}\left(X_{n}\right)$ follow from Lemma 3.5, while information about $\operatorname{Hg}\left(X_{n}\right)$ can be read off from [25, Section 2] (for $n=2,3$ and 5) and [24, 7.4(i), 7.5(i), 7.6(i)] (for $n=4)$.

If $n \leq 4$, then $\operatorname{Hg}(X) \cong \operatorname{Hg}\left(X_{0} \times X_{n}\right)$.

If $n=1, X_{1}$ is in fact isogenous to $X_{0}$ (but equipped with the conjugate action $\bar{\iota}$ of $\left.\mathcal{O}_{E}\right)$. Consequently, $\operatorname{Hg}\left(X_{0}^{4} \times X_{1}\right) \cong \operatorname{Hg}\left(X_{0}^{5}\right) \cong \operatorname{Hg}\left(X_{0}\right)$.

If $n=2$, then $\operatorname{Hg}(X) \cong \operatorname{Hg}\left(X_{0}\right) \times \operatorname{Hg}\left(X_{n}\right)$ ([25,3.2(ii)].

If $n=3$ or $n=4$, then $\operatorname{Hg}\left(X_{0} \times X_{n}\right)$ is calculated in [25, 5.3] and [25, 5.11], respectively.

If $n=5$, then $\operatorname{Hg}(X)=\operatorname{Hg}\left(X_{n}\right)$ has already been calculated.

Remark 3.8. We have phrased our answer in terms of Hodge groups, simply because one often has $\operatorname{Hg}(X \times Y) \cong \operatorname{Hg}(X) \times \operatorname{Hg}(Y)$, while this is never true for MumfordTate groups. Still, it is no more difficult to calculate $\operatorname{MT}(X)$. Recall that there is a $\mathbb{Q}$-linear determinant $\operatorname{det}_{\mathbb{Q}}: \operatorname{MT}(X) \rightarrow \mathbb{G}_{m}$, whose kernel is precisely $\operatorname{Hg}(X)$. For example, $\operatorname{MT}\left(X_{0}\right)$ is the torus $\mathbf{R}_{E / \mathbb{Q}} \mathbb{G}_{m}$, and in this case $\operatorname{det}_{\mathbb{Q}}$ coincides with the 
$E / \mathbb{Q}$-norm. In case (a) of Proposition 3.6, if $X_{1}$ has endomorphism ring $\mathbb{Z}$, then $\operatorname{MT}\left(X_{1}\right) \cong \mathrm{GL}_{2}$, and

$$
\operatorname{MT}(X) \cong\left\{(\alpha, \beta) \in E^{\times} \times \operatorname{GL}_{2}: \operatorname{det}_{\mathbb{Q}}(\alpha)=\operatorname{det}_{\mathbb{Q}}(\beta)\right\},
$$

while if $\operatorname{End}\left(X_{2}\right) \cong K$ an imaginary quadratic field, then

$$
\begin{aligned}
\operatorname{MT}(X) & \cong\left\{(\alpha, \beta) \in E^{\times} \times K^{\times}: \operatorname{det}_{\mathbb{Q}}(\alpha)=\operatorname{det}(\beta)\right\} \\
& =\left\{(\alpha, \beta) \in E^{\times} \times K^{\times}: \mathcal{N}_{E / \mathbb{Q}}(\alpha)=\mathcal{N}_{K / \mathbb{Q}}(\beta)\right\} .
\end{aligned}
$$

3.4. Endomorphism rings. Lemma 3.3 implies that an abelian fivefold with $\mathbb{Z}\left[\zeta_{3}\right]$ action of signature $(4,1)$ has at most one simple isogeny factor of dimension $n>1$. Lemma 3.5 shows that (if $n \neq 2$ ) then the endomorphism algebra of such a factor is the compositum of $\mathbb{Q}\left(\zeta_{3}\right)$ and $K_{0}$, a totally real field of degree $m$, where $m \mid n$. The goal of the present section is to show (Proposition 3.12) that every combination of $m$ and $n$ allowed by Lemmas 3.3 and 3.5 occurs for the abelian variety attached to some cubic surface.

The crux is to construct principally polarized abelian varieties with action by $\mathbb{Z}\left[\zeta_{3}\right]$, as opposed to a smaller order. (The fact that $\mathcal{N}=\tau(\mathcal{S})$ is open in $\mathcal{M}$ then implies that, possibly after an isogeny which still preserves the $\mathbb{Z}\left[\zeta_{3}\right]$-action and principal polarization, such an abelian variety is attached to a smooth cubic surface.) As noted in the introduction, in general it is delicate to adjust a polarized abelian variety in its isogeny class in order to simultaneously enlarge its endomorphism ring and induce a principal polarization. See [31] for a discussion of this problem.

In fact, it is no harder to work in a somewhat more general context than that narrowly required by Lemma 3.5. We thus introduce the following objects and notation, which will be in force throughout the present subsection. Let $K_{0}$ be a totally real field of degree $\left[K_{0}: \mathbb{Q}\right]=m$. To make progress on the task at hand, we will assume:

$K_{0}$ is a totally real field such that the inverse different $\mathcal{D}_{K_{0} / \mathbb{Q}}^{-1}$ is a square in the strict class group $\mathrm{Cl}^{+}\left(K_{0}\right)$ of $K_{0}$.

Note that this hypothesis is satisfied if the strict class group of $K_{0}$ has odd order, and in particular if it is trivial; a complete characterization of (3.4.1) may be found in [13, Proposition 3.1]. Fix an ordering of the distinct embeddings $\sigma_{1}, \ldots, \sigma_{m}: K_{0} \hookrightarrow \mathbb{R}$.

Now let $E$ be a quadratic imaginary field; fix an embedding $\iota: E \hookrightarrow \mathbb{C}$, and let $K$ be the compositum $K=K_{0} E$. All polarizations of abelian varieties with action by $\mathcal{O}_{K}$ considered in this section will be compatible with that action, in the sense that the Rosati involution induces complex conjugation on $\mathcal{O}_{K}$.

A CM type (for $K$ ) is a set $\Sigma$ of embeddings $\phi: K \rightarrow \mathbb{C}$ such that $\phi \in \Sigma$ if and only if $\bar{\phi} \notin \Sigma$, where - denotes complex conjugation. There is a bijection between subsets $S \subset\{1, \ldots, m\}$ and $\mathrm{CM}$ types for $K$. Indeed, let $\tau_{i}: K \hookrightarrow \mathbb{C}$ be the embedding such that $\left.\tau_{i}\right|_{E}=\iota$ and $\left.\tau_{i}\right|_{K_{0}}=\sigma_{i}$. Then define a CM type $\Sigma(S)$ by declaring that $\tau_{i} \in \Sigma(S)$ if and only if $i \in S$.

Note that if $X$ is an abelian variety with CM by $K$ of type $\Sigma(S)$, then it supports an $E$-action of signature $(\# S, m-\# S)$.

Lemma 3.9. Given $S \subset\{1, \ldots, m\}$, there is a principally polarized abelian variety $X$ of dimension $m$ equipped with an action by $\mathcal{O}_{K}$, compatible with the Rosati involution, such that $X$ has type $\Sigma(S)$. 
Proof. Record the CM type $\Sigma(S)$ as the ordered tuple $\phi_{1}, \ldots, \phi_{m}: K \hookrightarrow \mathbb{C}$, where $\left.\phi_{i}\right|_{K_{0}}=\sigma_{i}$. Then $K$ acts on $\mathbb{C}^{m}$ by

$$
a \cdot\left(t_{1}, \ldots, t_{m}\right)=\left(\phi_{1}(a) t_{1}, \ldots, \phi_{m}(a) t_{m}\right) .
$$

Recall the following classical construction of analytic families of HilbertBlumenthal abelian varieties (e.g., [12, Section 2.2]). Fix fractional ideals $\mathfrak{a}$ and $\mathfrak{b}$ of $K_{0}$. Given a point $\underline{z}=\left(z_{1}, \ldots, z_{m}\right)$ in the $m$-fold product of the upper half-plane $\mathbb{H}^{m}$, one defines a lattice $\Lambda_{\underline{z}} \subset \mathbb{C}^{m}$ by

$$
\begin{aligned}
\Lambda_{\underline{z}} & =\{\alpha \cdot \underline{z}+\beta \cdot \underline{1}: \alpha \in \mathfrak{a}, \beta \in \mathfrak{b}\} \\
& :=\left\{\left(\sigma_{1}(\alpha) z_{1}+\sigma_{1}(\beta), \cdots, \sigma_{m}(\alpha) z_{m}+\sigma_{m}(\beta)\right): \alpha \in \mathfrak{a}, \beta \in \mathfrak{b}\right\} .
\end{aligned}
$$

Then $X_{\underline{z}}=\mathbb{C}^{m} / \Lambda_{\underline{z}}$ is a complex analytic torus with an action by $\mathcal{O}_{K_{0}}$. For a fixed nonzero $\gamma \in\left(\mathcal{D}_{K_{0}} \mathfrak{a} \mathfrak{b}\right)^{-1} \subset K_{0}$, define a pairing

$$
\begin{aligned}
& (\mathfrak{a} \oplus \mathfrak{b}) \times(\mathfrak{a} \oplus \mathfrak{b}) \stackrel{E_{\gamma}}{\longrightarrow} \mathbb{Q} \\
& \left(\alpha_{1}, \beta_{1}\right) \times\left(\alpha_{2}, \beta_{2}\right) \longmapsto \operatorname{tr}_{K_{0} / \mathbb{Q}}\left(\gamma\left(\alpha_{1} \beta_{2}-\alpha_{2} \beta_{1}\right)\right) .
\end{aligned}
$$

Then $E_{\gamma}$ takes on values in $\mathbb{Z}$, and is the imaginary part of the Riemann form

$$
H_{\gamma, \underline{z}}\left(\left(x_{1}, \ldots, x_{m}\right),\left(y_{1}, \ldots, y_{m}\right)\right)=\sum_{1 \leq i \leq m} \frac{x_{i} \bar{y}_{i} \sigma_{i}(\gamma)}{\Im\left(z_{i}\right)},
$$

which is (the first Chern class of) an $\mathcal{O}_{K_{0}}$-linear polarization $\lambda_{\gamma}$ of $X_{\underline{z}}$. If one further assumes that $\mathfrak{a b}=\mathcal{D}_{K_{0}}^{-1}$ in $\mathrm{Cl}\left(K_{0}\right)^{+}$, then $\gamma$ may be chosen so that $\lambda_{\gamma}$ is principal.

We now specialize this construction to produce the desired $X$. Let $\mathfrak{a}=\mathfrak{b}$ be a fractional ideal in $K_{0}$ such that $\mathfrak{a} \cdot \mathfrak{a}$ is in the strict ideal class of $\mathcal{D}_{K_{0}}^{-1}$, and choose a $\gamma$ so that $H_{\gamma, \underline{z}}$ is a principal polarization on $\mathbb{C}^{m} / \Lambda_{\underline{z}}$ for each $\underline{z} \in \mathbb{H}^{m}$.

Fix a lattice $\mathbb{Z} \cdot 1 \oplus \mathbb{Z} \cdot z_{0} \subset \mathbb{C}$ which is stable under $\iota\left(\mathcal{O}_{E}\right)$. Let $\underline{z}^{*}=\left(z_{0}, \ldots, z_{0}\right)$, and let $X=\mathbb{C}^{m} / \Lambda_{\underline{z}^{*}}$. Then $H_{\gamma, \underline{z}^{*}}$ defines a principal polarization, and in particular $X$ is an abelian variety with an action by $\mathcal{O}_{K_{0}}$.

In fact, $X$ also supports an action by $\mathcal{O}_{E}$. To see this, suppose $\epsilon \in \mathcal{O}_{E}$. There are integers $a, b, c$ and $d$ such that

$$
\begin{aligned}
\epsilon \cdot 1 & =a z_{0}+b \\
\epsilon \cdot z_{0} & =c z_{0}+d .
\end{aligned}
$$

Given a lattice element $\alpha \cdot \underline{z}^{*}+\beta \cdot \underline{z}^{*} \in \Lambda_{\underline{z}^{*}}$, we then have

$$
\begin{aligned}
\epsilon \cdot\left(\alpha \cdot \underline{z}^{*}+\beta \cdot \underline{z}^{*}\right) & =\alpha \cdot\left(c \underline{z}^{*}+d \underline{1}\right)+\beta \cdot\left(a \underline{z}^{*}+b \underline{1}\right) \\
& =(c \alpha+a \beta) \underline{z}^{*}+(d \alpha+b \beta) \cdot \underline{1} \\
& \in \Lambda_{z^{*}}
\end{aligned}
$$

since $\alpha, \beta \in \mathfrak{a}$.

Moreover, the CM type of the action of $K$ is $\Sigma(S)$. (This is essentially worked out in [26, Section 22], albeit with a different, though commensurable, choice of lattice since the degree of the polarization is not germane there.) 
Now consider an abelian variety $Y$ of arbitrary dimension, equipped with an action by $\mathcal{O}_{K}$ such that $1 \in \mathcal{O}_{K}$ acts as $\operatorname{id}_{Y}$. The signature of this action - i.e., the isomorphism class of $\operatorname{Lie}(Y)$ as $\mathcal{O}_{K} \otimes \mathbb{C}$-module - is described by nonnegative integers $r_{i}$ and $s_{i}$ for $1 \leq i \leq m$. Here, $r_{i}$ is the dimension of the $\tau_{i}$-eigenspace of $\operatorname{Lie}(Y)$, and $s_{i}$ is the dimension of the $\bar{\tau}_{i}$-eigenspace. As an abelian variety with action by $\mathcal{O}_{E}, Y$ has signature $\left(\sum_{1 \leq i \leq m} r_{i}, \sum_{1 \leq i \leq m} s_{i}\right)$.

Lemma 3.10. Let $S^{(1)}, \ldots, S^{(n)}$ be subsets of $\{1, \ldots, m\}$.

(a) There is a principally polarized abelian variety $Y$ of dimension mn, equipped with an action by $\mathcal{O}_{K}$ of signature $\left(r_{1}, \ldots, r_{m} ; s_{1}, \ldots, s_{m}\right)$, where

$$
\begin{aligned}
& r_{i}=\#\left\{j: i \in S^{(j)}\right\} \\
& s_{i}=\#\left\{j: i \notin S^{(j)}\right\} .
\end{aligned}
$$

(b) Suppose there are indices $1 \leq i, j \leq m$ such that $r_{i} s_{i} \neq 0$ and $r_{j} s_{j} \neq 1$. Then there exists a $Y$ as in (a) such that $\operatorname{End}(Y) \cong \mathcal{O}_{K}$. In particular, such a $Y$ is simple.

Proof. For each $1 \leq j \leq m$, use Lemma 3.9 to construct a principally polarized abelian variety $X^{(j)}$ with action by $\mathcal{O}_{K}$ of signature $\Sigma\left(S^{(j)}\right)$. Then $Y=\times_{1 \leq j \leq n} X^{(j)}$, equipped with its product principal polarization, has an $\mathcal{O}_{K}$-action whose signature is given by (3.4.2). This proves (a).

Now use $H_{1}(Y, \mathbb{Z})$ and its Riemann form as input to Shimura's construction in [28, Section 2]. (The relevant case is also cleanly documented in [5, Section 9.6].) The output of this construction is a family of principally polarized abelian varieties on which $\mathcal{O}_{K}$ acts. The endomorphism ring of a sufficiently general member of this family is $\mathcal{O}_{K}$, and not larger, if the signature satisfies the combinatorial constraint given in (b) ([28, Theorem 5]; see also [5, Theorem 9.9.1]).

Lemma 3.11. Let $K_{0}$ be a totally real field of degree $m$ which satisfies (3.4.1). Let $E$ be a quadratic imaginary field and let $K=K_{0} E$. Given positive integers $n, r$ and $s$ satisfying $r+s=m n$, suppose that either:

(a) $n \geq 3$; or

(b) $n=2$ but either $m \geq 3$ or $m=2$ and $\{r, s\}=\{3,1\}$; or

(c) $n=1$ and $\operatorname{gcd}(r, s)=1$.

Then there exists a principally polarized abelian variety $Y$ of dimension $m n$ such that $\operatorname{End}(Y)=\mathcal{O}_{K}$ and the signature of $Y$, as $\mathcal{O}_{E}$-abelian variety, is $(r, s)$.

Proof. Given Lemma 3.10, it suffices to show that there exist subsets $S^{(1)}, \ldots, S^{(m)}$ such that, if $r_{i}$ and $s_{i}$ are calculated as in (3.4.2), then there are indices $i$ and $j$ such that $r_{i} s_{i} \neq 0$ and $r_{j} s_{j} \neq 1$. We briefly indicate how this may be accomplished. Note that if $S^{(j)}$ is represented as a row of length $m$ with entries + and -, then specifying types $S^{(1)}, \ldots, S^{(n)}$ is equivalent to labeling each cell of an $n \times m$ matrix with either + or - . Then $r_{i}$ is the number of + symbols in column $i$, and $s_{i}$ is defined analogously.

We may and do assume that $r \geq s$. Suppose $n \geq 3$. Any labeling of the $n \times m$ matrix in which both symbols appear in the first column will suffice, since then $r_{1}, s_{1} \geq 1$ and $r_{1}+s_{1}=n \geq 3$. This proves (a). 
Now suppose $n=2$. Under the additional hypotheses, $r \geq 3$ and $s \geq 1$. Consequently, we may choose a labeling in which the first two columns are given by

$$
\begin{aligned}
& ++ \\
& +-
\end{aligned}
$$

This proves (b).

For part (c), the hypothesis implies that any CM type $S$ with $\mathcal{O}_{E}$-signature $(r, s)$ is simple, and thus so is an associated abelian variety ([26, p. 213]).

If one is willing to believe the folklore conjecture that a positive proportion of totally real fields have strict class number one, there are many examples of the behavior allowed by Lemma 3.5:

Proposition 3.12. Let $K_{0}$ be a totally real field of degree $m$ which satisfies (3.4.1), and let $K=K_{0}\left(\zeta_{3}\right)$. Let $n$ be a natural number such that $m n \neq 2$.

(a) There exists a simple principally polarized abelian variety $X$ of dimension $m n$ such that $\operatorname{End}(X) \cong \mathcal{O}_{K}$ and, as $\mathbb{Z}\left[\zeta_{3}\right]$-abelian variety, $X$ has signature $(m n-1,1)$.

(b) If furthermore $1 \leq m n \leq 5$, then there exists a smooth cubic surface $Y$ for which the associated abelian variety $Q(Y)$ is isogenous to $X_{0}^{5-m n} \times X$, where $X_{0}$ is the elliptic curve with $\operatorname{End}\left(X_{0}\right) \cong \mathbb{Z}\left[\zeta_{3}\right]$.

Proof. Part (a) is a special case of Lemma 3.11. For part (b), consider the moduli point of $X_{0}^{5-m n} \times X$ in $\mathcal{M}(\mathbb{C})$. Its Hecke orbit is analytically dense in $\mathcal{M}_{\mathbb{C}}$, and in particular include points in the (Zariski open) subset $\tau\left(\mathcal{S}_{\mathbb{C}}\right)=\mathcal{N}_{\mathbb{C}}$.

Remark 3.13. Carlson and Toledo have also addressed the question of when an abelian variety with complex multiplication admits a principal polarization. In particular, [7, Proposition 5] proves a result similar to Proposition 3.12 in the special case $n=1$, albeit with an additional positivity condition on $\mathfrak{a}$ and less explicit control over $r$ and $s$.

For the sake of completeness (and at the suggestion of the referee), we observe that the case $g=2$ of Lemma 3.5 occurs, too.

Lemma 3.14. Let $D$ be an indefinite, nonsplit quaternion algebra over $\mathbb{Q}$ which contains $E$. Then there exists a principally polarized abelian surface $X$ with action by $\mathcal{O}_{E}$ such that $\operatorname{End}(X)_{\mathbb{Q}} \cong D$ and $X$, as $\mathcal{O}_{E}$-abelian variety, has signature $(1,1)$.

Proof. Let $D$ be an indefinite quaternion algebra over $\mathbb{Q}$ which contains $E$; choose an involution $(*)$ on $D$ which induces complex conjugation on $E$. Let $\mathcal{O}_{D}$ be any maximal order of $D$ which contains $\mathcal{O}_{E}$. There is a (one-dimensional, and in particular nonempty) Shimura curve whose points parametrize abelian surfaces $X$ with an action by $\mathcal{O}_{D}$. On each $X$ there exists a unique principal polarization for which the induced Rosati involution coincides with $(*)$ [6, Proposition III.1.5]. If $X$ is sufficiently general, then $\operatorname{End}(X)_{\mathbb{Q}}$ is $D$, and not larger (again, see [28, Theorem 5] or [5, Theorem 9.9.1]). It remains to verify the signature condition. By the Skolem-Noether theorem, there exists some $a \in D$ such that, for $b \in E \subset D, b^{(*)}=a b a^{-1}$. The action of $b$ on $\operatorname{Lie}(X)$ thus exchanges the two eigenspaces for the action of $\mathcal{O}_{E}$, and each is necessarily one-dimensional. 


\section{Arithmetic applications}

We start by calculating the geometric monodromy group of the first étale cohomology of the tautological abelian scheme over $\widetilde{\mathcal{T}}$ and its pullback to $\widetilde{\mathcal{S}}$. This is an arithmetic analogue of the topological monodromy calculation by Allcock et al. (Proposition 3.1). Subsequently, this is applied to two problems in the arithmetic of cubic surfaces.

First, we show that there are cubic surfaces $Y$ and threefolds $Z$ with rational coefficients for which $\operatorname{End}(Q(Y))$ and $\operatorname{End}(P(Z))$ are as small as possible.

Second, the arithmetic of the 27 lines on a cubic surface is a natural locus of inquiry [17]. We close the paper by calculating the field of definition of the lines on a cubic surface over a field of positive characteristic.

4.1. $\mathbb{Z}_{\ell}$-monodromy. There is a natural abelian scheme over $\tilde{\mathcal{T}}$, which pulls back to one on $\widetilde{\mathcal{S}}$. The first étale cohomology group of this abelian scheme gives a lisse

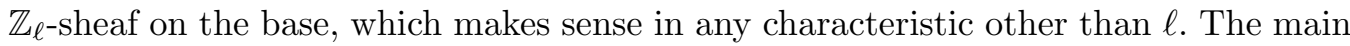
goal of the present section is to compute the geometric monodromy groups of these $\mathbb{Z}_{\ell}$ sheaves. (In characteristic zero, this recovers the fact (Proposition 3.1) that the connected component of the topological monodromy group of $\widetilde{\mathcal{S}}$ is $\mathrm{SU}$.)

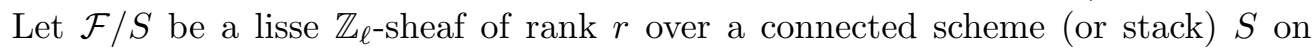
which $\ell$ is invertible. After choosing a geometric point $s \in S$, such an object is equivalent to a representation $\rho_{\mathcal{F} / S}: \pi_{1}(S, s) \rightarrow \operatorname{Aut}\left(\mathcal{F}_{s}\right) \cong \operatorname{GL}_{r}\left(\mathbb{Z}_{\ell}\right)$. Let $\mathrm{M}(\mathcal{F} / S)$ be the monodromy group of $\mathcal{F}$, i.e., the image of this representation; it is a closed subgroup of the $\ell$-adic group $\mathrm{GL}_{r}\left(\mathbb{Z}_{\ell}\right)$.

Recall that, if $\ell$ is an odd prime, if $H$ is either a special unitary group $\mathrm{SU}_{r, s}$ or symplectic group $\mathrm{Sp}_{2 g}$ and if $\Gamma \subset H\left(\mathbb{Z}_{\ell}\right)$ is a closed subgroup, then $\Gamma=H$ if and only if the composition $\Gamma \hookrightarrow H\left(\mathbb{Z}_{\ell}\right) \rightarrow H(\mathbb{Z} / \ell)$ is surjective. In this way, calculating the

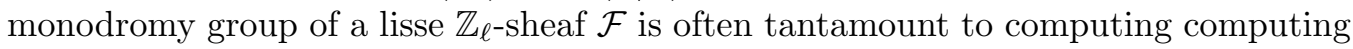
the monodromy group of the $\mathbb{Z} / \ell$-sheaf $\mathcal{F} \otimes \mathbb{Z} / \ell$.

We start with some geometry. Let $\mathcal{H}_{5}$ be the moduli space of hyperelliptic curves of genus five, identified with its image in $\mathcal{A}_{5}$ under the (classical) Torelli map.

Proposition 4.1. Let $k$ be a field in which 2 is invertible. Then the closure of $\widetilde{\varpi}\left(\widetilde{\mathcal{T}}_{k}\right)$ in $\mathcal{A}_{5, k}$ contains $\mathcal{H}_{5, k}$.

Proof. Since taking Zariski closure commutes with flat base change, we assume that $k$ is algebraically closed. Let $X / k$ be an abelian fivefold which is a hyperelliptic Jacobian. More explicitly, suppose $X$ is the Jacobian of the hyperelliptic curve branched at points $q_{1}, \ldots, q_{12} \in \mathbb{P}^{1}$. Identify this copy of $\mathbb{P}^{1}$ with a rational normal curve $N \subset \mathbb{P}^{4}$, and let $Z_{0}$ be the secant variety of $N$. Then $Z_{0}$ is a cubic threefold, singular exactly along $N$. Let $G_{0}$ be a defining cubic form for this singular threefold. Let $Z_{1}$ be any smooth cubic threefold passing through each of the $p_{i}$, transverse to $N$, and let $G_{1}$ be a defining cubic form for $Z_{1}$.

Consider the cubic threefold $Z$ over $k \llbracket t \rrbracket$ with defining equation $t^{2} G_{1}+G_{0}$. To prove the proposition, it suffices to show that the closure of $P\left(Z_{k((t))}\right)$ in $\mathcal{A}_{5}$ contains the moduli point of $X$.

If $k$ has characteristic zero, this is the main result of [8]; the claim is proved by transcendental means. 
If $k$ has positive characteristic, let $R$ be a mixed characteristic discrete valuation ring with residue field $k$, and let $K=$ Frac $R$ be its fraction field. Lift $N$ to a rational normal curve $\hat{N} \subset \mathbb{P}_{R}^{4}$, and for $i \in\{1, \ldots, 12\}$ let $\hat{q}_{i} \in \hat{N}(R)$ be a section lifting $q_{i}$. Let $\hat{Z}_{0} / R$ be the secant variety of $\hat{N}$. Similarly, let $\hat{Z}_{1} / R$ lift $Z_{1}$ and pass through each $\hat{q}_{i}$ transversally to $\hat{N}$. For $i=1,2$, let $\hat{G}_{i}$ be the cubic form with coefficients in $R$ defining $\hat{Z}_{i}$, and consider the cubic form $t^{2} \hat{G}_{1}+\hat{G}_{0}$ over $R \llbracket t \rrbracket$.

Let $\xi$ : Spec $R \llbracket t \rrbracket \hookrightarrow \overline{\mathcal{A}}_{5}$ be the closure of the moduli point of $P\left(Z_{K((t))}\right)$ in the minimal compactification of $\mathcal{A}_{5}$. The result in characteristic zero shows that $\xi_{K}$ : Spec $K \rightarrow$ Spec $K \llbracket t \rrbracket \hookrightarrow \overline{\mathcal{A}}_{5}$ is the moduli point of the Jacobian $\hat{X}_{K}$ of the hyperelliptic curve branched over (the generic fibers of) $\hat{q}_{1}, \ldots, \hat{q}_{12}$. Since the closure $\xi_{R}$ of $\xi_{K}$ contains the moduli point of $X, \xi$ is in fact a morphism $\xi: \operatorname{Spec} R \llbracket t \rrbracket \hookrightarrow \mathcal{A}_{5}$. Moreover, $\xi_{k}$ is in the closure of $\xi_{k((t))}$, which means that $P\left(Z_{k((t))}\right)$ is the generic fiber of a deformation of $X$.

The next result says that certain natural monodromy groups attached to cubic surfaces and threefolds are as large as possible, given the obvious constraints imposed by polarization and, for cubic surfaces, an action by $\mathbb{Z}\left[\zeta_{3}\right]$.

Remark 4.2. The $\overline{\mathbb{Q}}_{\ell}$-monodromy group of the universal family of hypersurfaces of given degree and dimension is known, thanks to work of Deligne [10, 4.4.1]. The

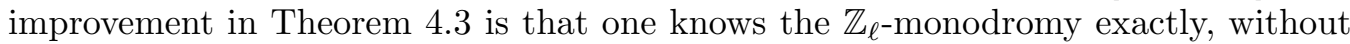
passage to the Zariski closure or extension to $\overline{\mathbb{Q}}_{\ell}$-coefficients.

Recall that the tautological abelian fivefold and cubic threefold are given by $f$ : $\mathcal{X} \rightarrow \mathcal{A}_{5}$ and $h: \mathcal{Z} \rightarrow \tilde{\mathcal{T}}$.

Theorem 4.3. Let $k$ be an algebraically closed field in which 2 is invertible, and let $\ell$ be an odd rational prime invertible in $k$. Then:

(a) $\mathrm{M}\left(R^{1} f_{*} \mathbb{Z}_{\ell} \rightarrow \widetilde{\varpi}\left(\widetilde{\mathcal{T}}_{k}\right)\right) \cong \operatorname{Sp}_{10}\left(\mathbb{Z}_{\ell}\right)$;

(b) $\mathrm{M}\left(R^{3} h_{*} \mathbb{Z}_{\ell}(1) \rightarrow \widetilde{\mathcal{T}}\right) \cong \operatorname{Sp}_{10}\left(\mathbb{Z}_{\ell}\right)$;

(c) $\mathrm{M}\left(\widetilde{\phi}^{*} R^{3} h_{*} \mathbb{Z}_{\ell}(1) \rightarrow \widetilde{\mathcal{S}}\right) \cong \mathrm{U}^{*}\left(\mathbb{Z}_{\ell}\right)$ if $\ell \neq 3$ and $\operatorname{char}(k) \neq 3$.

Proof. For (a), if $S \subset \mathcal{A}_{5, k}$ is any subspace, there is an a priori constraint $\mathrm{M}\left(R^{1} f_{*} \mathbb{Z}_{\ell} \rightarrow\right.$ $S) \subset \mathrm{Sp}_{10}\left(\mathbb{Z}_{\ell}\right)$. By semicontinuity and Proposition $4.1, \mathrm{M}\left(R^{1} f_{*} \mathbb{Z}_{\ell} \rightarrow \widetilde{\varpi}\left(\widetilde{\mathcal{T}}_{k}\right)\right)$ contains $\mathrm{M}\left(R^{1} f_{*} \mathbb{Z}_{\ell} \rightarrow \mathcal{H}_{5}\right)$. This last group is known to be $\mathrm{Sp}_{10}\left(\mathbb{Z}_{\ell}\right)$ (e.g., [2, Theorem 3.4], $[15])$.

Part (b) then follows from the isomorphism $\widetilde{\varpi}^{*} R^{1} f_{*} \mathbb{Z}_{\ell} \rightarrow R^{3} h_{*} \mathbb{Z}_{\ell}(1)$ established in $[1$, Proposition 3.6] and the fact that $\widetilde{\varpi}$ is a fibration.

For part (c), the assertion that $\mathrm{M}\left(R^{1} f_{*} \mathbb{Z}_{\ell} \rightarrow \mathcal{M}\right) \cong \mathrm{U}^{*}\left(\mathbb{Z}_{\ell}\right)$ follows from the transcendental uniformization of $\mathcal{M}_{\mathbb{C}}$ and the theory of arithmetic compactifications; the details are provided in Section 4.2, and in particular Lemma 4.5.

Since $\tau: \mathcal{S}_{k} \rightarrow \mathcal{M}_{k}$ is an open embedding, each irreducible component of the PEL Shimura variety $\mathcal{M}_{k}^{\ell}$ analyzed in Lemma 4.5 pulls back to an irreducible cover of $\mathcal{S}_{k}$, and in particular $\mathrm{M}\left(\widetilde{\phi}^{*} R^{3} h_{*} \mathbb{Z}_{\ell}(1) \rightarrow \mathcal{S}\right) \cong \mathrm{U}^{*}\left(\mathbb{Z}_{\ell}\right)$. Now use the fact that $\widetilde{S} \rightarrow S$ is a fibration with connected fibers. 
4.2. Monodromy groups for unitary Shimura varieties. The goal of this section is to calculate the $\ell$-adic monodromy for a unitary Shimura variety attached to $\mathbb{Z}\left[\zeta_{3}\right]$. It is no harder to allow an essentially arbitrary signature condition on the $\mathbb{Z}\left[\zeta_{3}\right]$-action, and this is useful for applications outside the present paper [2].

Some notation and background on unitary groups will be useful. For positive integers $r$ and $s$, let $g=r+s$ and let $V_{r, s}$ be the module $\mathcal{O}_{E}^{\oplus g}$, with standard basis $e_{1}, \ldots, e_{g}$. Endow $V_{r, s}$ with the Hermitian pairing

$$
\left\langle\left\langle\sum x_{j} e_{j}, \sum y_{j} e_{j}\right\rangle\right\rangle=\sum_{1 \leq j \leq r} x_{j} \bar{y}_{j}-\sum_{r+1 \leq j \leq g} x_{j} \bar{y}_{j} .
$$

As in Section 2.2, define $\mathrm{GU}_{r, s}=\mathrm{GU}\left(V_{r, s},\langle\langle\cdot, \cdot\rangle\rangle\right)$, and define $\mathrm{SU}_{r, s}$ and $\mathrm{U}_{r, s}$ analogously. If $\mathrm{T}$ and $\mathrm{T}_{1}$ are the tori defined in (2.2.1)-(2.2.2), then these group schemes again sit in exact sequences (2.2.3). Finally, as in (2.2.4), let $\mathrm{U}_{r, s}^{*}$ be the extension of the finite group scheme $\boldsymbol{\mu}_{6}$ by $\mathrm{SU}_{r, s}$, so that $\mathrm{U}_{r, s}^{*}(\mathbb{Z} / N)$ is the extension of the global units $\mathcal{O}_{E}^{\times}$by $\mathrm{SU}_{r, s}(\mathbb{Z} / N)$.

If $\ell$ is inert in $\mathcal{O}_{E}$, then $\mathrm{SU}_{r, s}(\mathbb{Z} / \ell)$ is isomorphic to the classical group $\operatorname{SU}\left(g, \mathbb{F}_{\ell}\right)$, while if $\ell$ splits, then $\mathrm{SU}_{r, s}(\mathbb{Z} / \ell) \cong \operatorname{SL}\left(g, \mathbb{F}_{\ell}\right)$.

Lemma 4.4. Let $\ell$ be a prime not equal to 3 . The natural map $\mathrm{SU}_{r, s}(\mathbb{Z}) \rightarrow \mathrm{SU}_{r, s}(\mathbb{Z} / \ell)$ is surjective.

Proof. This is presumably well-known. For want of a reference, we provide a proof here, proceeding by induction on $r+s$.

Suppose $r=s=1$. Note that, for any $\operatorname{ring} R$, an element $v_{1} e_{1}+v_{2} e_{2} \in V \otimes R$ is isotropic if and only if $v_{2}$ is either $v_{1}$ or $\bar{v}_{1}$. Now, $\mathrm{SU}_{1,1}(\mathbb{Z} / \ell)$ is generated by isotropic transvections, i.e., maps of the form

$$
\tau_{u, a}: v \longmapsto a\langle\langle v, u\rangle\rangle u
$$

where $u \in V \otimes \mathbb{Z} / \ell$ is isotropic and $a \in \mathcal{O}_{E} / \ell$ satisfies $a=-\bar{a}$. (This is well-known if $\mathcal{O}_{E} / \ell \cong \mathbb{F}_{\ell^{2}}$ is a field; if $\ell$ splits in $\mathcal{O}_{E}$, this is is still true, since the quotient ring is semilocal [14, p. 535].) It is clear that such a $u$ admits an isotropic lift $\widetilde{u}$ to $V$ itself. Similarly, let $\widetilde{a} \in \mathcal{O}_{E}$ be a totally imaginary element (i.e., $\widetilde{a}+\overline{\widetilde{a}}=0$ ) whose reduction modulo $\ell$ is $a$. Then

$$
\widetilde{\tau}_{\widetilde{u}, \widetilde{a}}: v \longmapsto \widetilde{a}\langle\langle v, \widetilde{u}\rangle\rangle \widetilde{u}
$$

is an element of $\mathrm{SU}\left(V_{1,1}\right)$ whose reduction modulo $\ell$ is $\tau_{u, a}$. Thus, the image of $\mathrm{SU}_{1,1}(\mathbb{Z}) \rightarrow \mathrm{SU}_{1,1}(\mathbb{Z} / \ell)$ contains a generating set for the range.

Now suppose the claim is known in dimension $g-1$. We may and do suppose

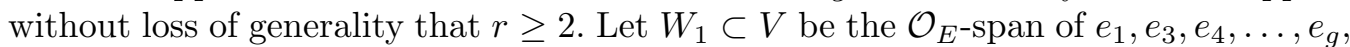
and let $W_{2}$ be the $\mathcal{O}_{E}$-span of $e_{2}, e_{3}, \ldots, e_{g}$. Then $\mathrm{SU}\left(W_{i}\right) \cong \mathrm{SU}_{r-1, s}$; by induction, $\mathrm{SU}\left(W_{i}\right)(\mathbb{Z})$ surjects onto $\mathrm{SU}\left(W_{i}\right)(\mathbb{Z} / \ell)$. It is not hard to check (e.g., [2, Lemma 3.2]) that, independent of the splitting behavior of $\ell$ in $\mathcal{O}_{E}$, the only subgroup of $\mathrm{SU}_{r, s}(\mathbb{Z} / \ell)$ which contains each $\mathrm{SU}\left(W_{i}\right)(\mathbb{Z} / \ell)$ is $\mathrm{SU}_{r, s}(\mathbb{Z} / \ell)$ itself.

To ease notation, we now fix positive integers $r$ and $s$, and write $\mathrm{SU}$ for $\mathrm{SU}_{r, s}$, etc. Let $\mathcal{M}=\mathcal{M}_{\mathbb{Z}\left[\zeta_{3}, 1 / 3\right](r, s)}$ be the moduli stack over $\mathbb{Z}\left[\zeta_{3}, 1 / 3\right]$ of triples $(X, \iota, \lambda)$ where $X$ is an abelian scheme of dimension $g=r+s ; \iota: \mathbb{Z}\left[\zeta_{3}\right] \hookrightarrow \operatorname{End}(X)$ is an action with signature $(r, s)$; and $\lambda$ is a principal polarization compatible with $\iota$. Of course, the datum $(r, s)=(4,1)$ is the one relevant, via $\tau$, to the study of cubic surfaces. 
Lemma 4.5. Let $k$ be an algebraically closed field in which 3 is invertible, and let $\ell \geq 5$ be a rational prime invertible in $k$. Let $t \in \mathcal{M}(k)$ be a geometric point. Then the image of the monodromy representation

$$
\pi_{1}\left(\mathcal{M}_{k}, t\right) \longrightarrow \operatorname{Aut}\left(\mathcal{X}_{t}[\ell]\right)
$$

is $\mathrm{U}^{*}(\mathbb{Z} / \ell)$.

Remark 4.6. Suppose that $\frac{g-1}{3} \leq r, s \leq \frac{2 g+1}{3}$. Then some $\mathbb{Z}\left[\zeta_{3}\right]$-abelian varieties of signature $(r, s)$ are actually Jacobians of cyclic triple covers of the projective line [2, Lemma 2.9]. Lemma 4.5 is compatible with [18, Theorem 5.4] but not, alas, [2, Theorem 3.8]; in spite of its claims to the contrary, [2] only calculates the connected component of the monodromy group.

Proof. We wish to describe the action of $\pi_{1}\left(\mathcal{M}_{k}, t\right)$ on $\mathcal{X}_{t}[\ell]$. Equivalently, we wish to describe the action of $\pi_{1}\left(\mathcal{M}_{k}, t\right)$ on the set of principal level $\ell$ structures on $\mathcal{X}_{t}[\ell]$. A principal level $\ell$ structure on a polarized abelian scheme with $\mathcal{O}_{E}$-action $(X / S, \iota, \lambda) \in$ $\mathcal{M}(S)$ is the data of an $\mathcal{O}_{E}$-equivariant isomorphism $\alpha:\left(V \otimes \mathcal{O}_{E} / \ell\right)_{S} \stackrel{\sim}{\rightarrow} X[\ell]$ and an isomorphism $\nu: \mathbb{Z} / \ell(1)_{S} \stackrel{\sim}{\rightarrow} \boldsymbol{\mu}_{\ell, S}$ such that the following diagram commutes:

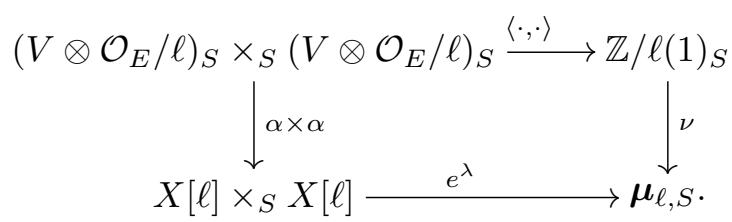

(Actually, we have chosen and then suppressed an isomorphism $\mathbb{Z} \stackrel{\sim}{\rightarrow} \mathbb{Z}(1)$, so that $\langle\cdot, \cdot\rangle$ takes values in $\mathbb{Z}(1)$.) This formulation of level structure is taken from [20, 1.3.6.1]; it is equivalent $[20,1.4 .3 .4]$ to the perhaps more frequent "rational level structure on abelian varieties up to isogeny" used in, e.g., [19], and more convenient for the application here.

Let $\mathcal{M}^{\ell}$ be the moduli space of principally polarized abelian varieties with $\mathbb{Z}\left[\zeta_{3}\right]$ structure of signature $(r, s)$ and principal level $\ell$ structure. Then, away from characteristic $\ell, \mathcal{M}^{\ell}$ is a $\mathrm{GU}(\mathbb{Z} / \ell)$-torsor over $\mathcal{M}$. (In fact, the choice of an isomorphism $\alpha$ determines the isomorphism $\nu$; but explicitly tracking both pieces of information makes it clear that $\mathcal{M}^{\ell}$ really is a $\mathrm{GU}(\mathbb{Z} / \ell)$-torsor.)

Let $t=(X, \iota, \lambda) \in \mathcal{M}(k)$, and let $\widetilde{t}=(X, \iota, \lambda,(\alpha, \nu)) \in \mathcal{M}^{\ell}(k)$ be a point lying over it. The action of $\pi_{1}\left(\mathcal{M}_{k}, t\right)$ on $\mathcal{X}_{t}[\ell]$ is the action of $\pi_{1}\left(\mathcal{M}_{k}, t\right)$ on $\tilde{t}$. In particular, the mod- $\ell$ monodromy group is (noncanonically) isomorphic to the group of automorphisms of an irreducible component of $\mathcal{M}_{k}^{\ell}$ over $\mathcal{M}_{k}$. Thanks to the existence of arithmetic toroidal compactifications [20,6.4.1.1] and the usual Zariski connectedness argument (e.g., $[20,6.4 .12])$, it suffices to carry out this last calculation in the special case $k=\mathbb{C}$.

There is a double quotient of the adèlic space $\mathrm{GU}(\mathbb{A})$ which encodes the structure of the set of components of $\mathcal{M}_{\mathbb{C}}^{\ell}$. This is in $[9,2.9]$, but we follow here the more expansive treatment in [22, Section 5].

Let $\mathbb{A}_{\text {fin }}$ denote the ring of finite adèles, and let $\mathrm{K}_{\ell}=\{\alpha \in G(\hat{\mathbb{Z}}): \alpha \equiv \operatorname{id} \bmod \ell\} \subset$ $G\left(\mathbb{A}_{\text {fin }}\right)$. Then $\mathcal{M}_{\mathbb{C}}^{\ell}$ admits the uniformization

$$
\mathcal{M}_{\mathbb{C}}^{\ell}=\mathrm{GU}(\mathbb{Q}) \backslash \mathbb{X} \times \mathrm{GU}\left(\mathbb{A}_{\text {fin }}\right) / \mathrm{K}_{\ell},
$$


where the Hermitian symmetric domain $\mathbb{X}$ is a certain $G U(\mathbb{R})$-conjugacy class of homomorphisms $\mathbf{R}_{\mathbb{C} / \mathbb{R}} \mathbb{G}_{m, \mathbb{C}} \rightarrow \mathrm{GU}_{\mathbb{R}}$.

Now, $\mathrm{T}$ is the maximal abelian quotient of $\mathrm{GU}, \mathrm{T}(\mathbb{R}) \cong \mathbb{C}^{\times}$is (topologically) connected, and SU is simply connected. Consequently, there is a canonical bijection between irreducible components of $\mathcal{M}_{\mathbb{C}}^{\ell}$ and the finite group

$$
\mathrm{T}(\mathbb{Q}) \backslash \mathrm{T}\left(\mathbb{A}_{\mathrm{fin}}\right) / \nu\left(\mathrm{K}_{\ell}\right)=E^{\times} \backslash \mathbb{A}_{E, \text { fin }}^{\times} / \nu\left(\mathrm{K}_{\ell}\right)
$$

$[22,5.17$ and p.311].

We compute this last quotient. The compact group $\nu\left(\mathrm{K}_{\ell}\right)$ is simply the group of finite idèles in $E$ which are everywhere integral and congruent to one modulo $\ell$. Indeed, let $\mathfrak{l}$ be the modulus associated to $\ell$; then $\nu\left(\mathrm{K}_{\ell}\right)$ is the finite part of the congruence group $\mathbb{A}_{E, l}^{\times}$. We thus recognize the quotient in (4.2.1) as

$$
\mathrm{Cl}_{E}^{\mathfrak{l}}=\frac{\mathbb{A}_{E}^{\times} / E^{\times}}{E^{\times} \mathbb{A}_{E, \mathfrak{l}}^{\times} / E^{\times}},
$$

the ray class group modulo $\mathfrak{l}$. The ray class group may, of course, be computed via ideals, instead of idèles. Since $E$ is totally imaginary, $\mathrm{Cl}_{E}^{\mathfrak{l}}$ fits into an exact sequence [23, Theorem 5.1.7]

$$
0 \longrightarrow \mathcal{O}_{E}^{\times} / \mathcal{O}_{E, \mathfrak{l}}^{\times} \longrightarrow\left(\mathcal{O}_{K} / \ell\right)^{\times} \longrightarrow \mathrm{Cl}_{E}^{\mathrm{l}} \longrightarrow \mathrm{Cl}_{E} \longrightarrow 0,
$$

where $\mathrm{Cl}_{E}$ is the class group of $E$ and $\mathcal{O}_{E, \mathfrak{l}}^{\times}$is the group of global units which are one modulo $\ell$. These two groups are trivial because, respectively, $\mathbb{Z}\left[\zeta_{3}\right]$ has unique factorization and $\ell \geq 5$. Consequently, $\mathrm{Cl}_{E}^{\mathfrak{l}} \cong\left(\mathcal{O}_{E} / \ell\right)^{\times} / \mathcal{O}_{E}^{\times}$.

In particular, the irreducible components of $\mathcal{M}_{\mathbb{C}}^{\ell}$ are parametrized by the cosets $\mathrm{GU}(\mathbb{Z} / \ell) / \mathrm{U}^{*}(\mathbb{Z} / \ell)$. Therefore, the stabilizer of each component of $\mathcal{M}_{\mathbb{C}}^{\ell}$ is $\mathrm{U}^{*}(\mathbb{Z} / \ell)$, as claimed.

4.3. Small endomorphism rings over $\mathbb{Q}$. Carlson and Toledo have given explicit examples of cubic surfaces $Y / K$ over number fields such that $\operatorname{End}(Q(Y))$ is large. The following result shows that the generic (i.e., small) endomorphism ring is also attainable over number fields. Note that Corollary 4.7 is not an immediate consequence of Lemma 3.2. If $T$ is a variety over $\mathbb{Q}$ and $\mathbb{W} \rightarrow T_{\mathbb{C}}$ is a variation of Hodge structure, cardinality considerations alone do not exclude the possibility that the Hodge-generic locus of $\mathbb{W} \rightarrow T_{\mathbb{C}}$ avoids all of $T(\overline{\mathbb{Q}})$.

Corollary 4.7. (a) There exists a cubic threefold $Z / \mathbb{Q}$ such that $\operatorname{End}_{\overline{\mathbb{Q}}}$ $(P(Z)) \cong \mathbb{Z}$.

(b) There exists a cubic surface $Y / \mathbb{Q}$ such that $\operatorname{End}_{\overline{\mathbb{Q}}}(Q(Y)) \cong \mathbb{Z}\left[\zeta_{3}\right]$.

Proof. This essentially follows from Theorem 4.3 and a result of Terasoma [30, Theorem 2]. Full details are provided here for part (b); part (a) is entirely analogous.

Fix a rational prime $\ell \geq 5$ which is inert in $\mathbb{Q}\left(\zeta_{3}\right)$. As in Section 2.2 , let $\mathcal{M}^{\ell}$ be the moduli space of principally polarized abelian varieties with $\mathbb{Z}\left[\zeta_{3}\right]$-structure of signature $(4,1)$ and principal level $\ell$ structure. Let $K$ be any number field such that every irreducible component of $\mathcal{M}_{K}^{\ell}$ is geometrically irreducible, and let $\mathcal{M}_{K}^{\ell, 0}$ be one such component. 
Let $\widetilde{\mathcal{M}}_{K}^{\ell, 0}$ be the pullback of $\mathcal{M}_{K}^{\ell, 0}$ to $\widetilde{\mathcal{S}}_{K}$. Since $\tau$ is an open immersion and $\widetilde{\mathcal{S}} \rightarrow \mathcal{S}$ is a fibration, $\widetilde{\mathcal{M}}_{K}^{\ell, 0} \rightarrow \widetilde{\mathcal{S}}$ is an absolutely irreducible Galois cover of varieties, with Galois group $U^{*}(\mathbb{Z} / \ell)$ (Lemma 4.5). Since $\widetilde{\mathcal{S}}_{K}$ is an open subset of a projective space, by Hilbert's irreducibility theorem [27, Proposition 3.3.1] there is a thin set $A \subset \widetilde{\mathcal{S}}(K)$ such that if $s \in \widetilde{\mathcal{S}}(K) \backslash A$, then $\widetilde{\mathcal{M}}_{s}^{\ell, 0} \rightarrow s \cong$ Spec $K$ is again irreducible.

For such an $s$, the absolute endomorphism ring of $Q\left(\mathcal{Y}_{s}\right)$ is $\mathbb{Z}\left[\zeta_{3}\right]$. Indeed, the image of the Galois representation $\pi_{1}(s, \bar{s}) \cong \operatorname{Gal}(K) \rightarrow \operatorname{Aut}\left(T_{\ell} Q\left(\mathcal{Y}_{s}\right)\right)$ contains $\mathrm{SU}\left(\mathbb{Z}_{\ell}\right)$, and thus for any finite extension $L / K$, the image of $\operatorname{Gal}(L)$ contains an open subgroup of $\mathrm{SU}\left(\mathbb{Z}_{\ell}\right)$. Since the $\ell$-adic completion of the endomorphism ring of $Q\left(\mathcal{Y}_{s}\right)_{L}$ is the commutant of the image of $\operatorname{Gal}(L)$, it follows that $\operatorname{End}_{L}\left(Q\left(\mathcal{Y}_{s}\right)\right) \cong \mathbb{Z}\left[\zeta_{3}\right]$.

Moreover, $A \cap \widetilde{\mathcal{S}}(\mathbb{Q})$ is also thin in $\widetilde{\mathcal{S}}(\mathbb{Q})[27$, Proposition 3.2.1], so there are many suitable cubic surfaces defined over $\mathbb{Q}$.

4.4. The Galois group of the 27 lines. Camille Jordan investigated ([17, III.II.V]; see also [16]) the structure of the field of definition of the 27 lines on a generic cubic surface in characteristic zero. His findings may be expressed in moduli-theoretic terms, as follows.

As in [1, Section 2.1] and [21, Section 2], a marking on a cubic surface $Y$ is an identification $\Psi$ of the configuration of lines on $Y$ (i.e., the 27 lines and their incidence relations) with an abstract configuration of this type. The set of all markings on a fixed cubic surface is a torsor under $W\left(\mathrm{E}_{6}\right)$, the Weyl group of the exceptional root system $E_{6}$. Let $\mathcal{S}^{\mathrm{m}} \rightarrow \operatorname{Spec} \mathcal{O}_{E}[1 / 6]$ be the moduli space (actually, a fine moduli scheme) of marked cubic surfaces. For a field $K$ equipped with a map $\mathcal{O}_{E}[1 / 6] \rightarrow K$, let $K\left(\mathcal{C}^{\mathrm{m}}\right)$ be the ring of rational functions on $\mathcal{S}^{\mathrm{m}} \times \operatorname{Spec} K$; similarly, let $K(\mathcal{S})$ be the ring of rational functions on $\underline{\mathcal{S}} \times \operatorname{Spec} K$, where $\underline{\mathcal{S}}$ is the coarse moduli scheme of the stack $\mathcal{S}$.

Jordan's results, in modern language, show that $\mathcal{S}_{\mathbb{C}}^{\mathrm{m}}$ is irreducible, and $\mathbb{C}\left(\mathcal{S}^{\mathrm{m}}\right) / \mathbb{C}(\mathcal{S})$ is a Galois extension of fields with Galois group $W\left(\mathrm{E}_{6}\right)$. In fact, this is true if $\mathbb{C}$ is replaced with an arbitrary field, and in particular one of positive characteristic.

For a prime $\mathfrak{p} \in \mathcal{O}_{E}[1 / 6]$, let $\kappa(\mathfrak{p})$ be its residue field.

Proposition 4.8. $\quad$ (a) Each fiber of $\mathcal{S}^{\mathrm{m}} \rightarrow \operatorname{Spec} \mathcal{O}_{E}[1 / 6]$ is geometrically irreducible.

(b) For $[\mathfrak{p}] \in \operatorname{Spec} \mathcal{O}_{E}[1 / 6], \mathcal{S}^{\mathrm{m}} \times[\mathfrak{p}]$ is geometrically irreducible, and $\kappa(\mathfrak{p})\left(\mathcal{S}^{\mathrm{m}}\right) /$ $\kappa(\mathfrak{p})(\mathcal{S})$ is a Galois extension of fields with group $W\left(\mathrm{E}_{6}\right)$.

Proof. Part (b) is a reformulation of part (a) which, like Lemma 4.5, is a consequence of a good theory of arithmetic compactification of PEL Shimura varieties. Indeed, let $\mathcal{M}^{\left(1-\zeta_{3}\right)} \rightarrow \operatorname{Spec} \mathbb{Z}\left[\zeta_{3}, 1 / 6\right]$ be the moduli space of principally polarized abelian $\mathbb{Z}\left[\zeta_{3}\right]$-fivefolds with full $\left(1-\zeta_{3}\right)$-level structure, i.e., with a rigidification of the kernel of multiplication by $1-\zeta_{3}$; see $\left[1\right.$, Section 2.2] for more details. Then $\mathcal{M}^{\left(1-\zeta_{3}\right)} \rightarrow \mathcal{M}$ is a $W\left(\mathrm{E}_{6}\right)$-torsor. Moreover, let $\mathcal{N}^{\left(1-\zeta_{3}\right)}=\mathcal{N} \times_{\text {Spec } M} \mathcal{M}^{\left(1-\zeta_{3}\right)}$; there is an isomorphism of fine moduli schemes $\mathcal{S}^{\mathrm{m}} \stackrel{\sim}{\rightarrow} \mathcal{N}^{\left(1-\zeta_{3}\right)}$ [1, Theorem 5.7].

In particular, for an algebraically closed field $K$ equipped with a morphism $\mathcal{O}_{E}[1 / 6] \rightarrow K$, there is a canonical bijection between the irreducible components of $\mathcal{S}_{K}^{\mathrm{m}}$ and of $\mathcal{N}_{K}^{\left(1-\zeta_{3}\right)}$. Since $\mathcal{N} \rightarrow \mathcal{M}$ is fiberwise an open, dense immersion, each of these is in bijection with the irreducible components of $\mathcal{M}_{K}^{\left(1-\zeta_{3}\right)}$. Finally, the existence 
of smooth compactifications [20,6.4.1.1] implies that the irreducible components of $\mathcal{M}_{K}^{\left(1-\zeta_{3}\right)}$ are in bijection with those of $\mathcal{M}_{\mathbb{C}}^{\left(1-\zeta_{3}\right)}$. The desired result now follows from Jordan's theorem.

Thus, if $K$ is a Hilbertian field (in which 6 is invertible), then for most cubic surfaces $Y / K$, the field of definition $K\left(\Psi_{Y}\right)$ of the 27 lines on $Y$ is as large as possible; $\operatorname{Gal}\left(K\left(\Psi_{Y}\right) / K\right) \cong W\left(\mathrm{E}_{6}\right)$. In particular, $\operatorname{Gal}(K)$ acts as the full automorphism group of the configuration of 27 lines.

Nonetheless, using the theory of complex multiplication, we are able to identify a class of cubic surfaces for which $\mathrm{Gal}(K)$ acts on $\Psi_{Y}$ via an abelian quotient. For example, suppose $Y \in \mathcal{S}(\mathbb{C})$ is special, i.e., that the Mumford-Tate group $\operatorname{MT}(X)$ of $X=Q(Y)$ is a torus. Then $X$ is an abelian variety of CM type, and is defined over a totally imaginary number field $K$. The Torelli map is an isomorphism of stacks over $E$, and so $Y$ is also defined over $K$. Let $[Y, \Psi] \in \mathcal{S}(\bar{K})$ be the moduli point of a marking of the cubic surface $Y$, and let $[X, \Phi]=\tau^{\mathrm{m}}([Y, \Psi])$. Then $K(\Psi) \cong K(\Phi)$ is the field obtained by adjoining the coordinates of all $\left(1-\zeta_{3}\right)$-torsion of $X$ to $K$, and thus is an abelian extension of $K$.

Moreover, the reciprocity law attached to the Shimura variety $\mathcal{M}^{\mathrm{m}}[9]$ explicitly describes the action of $\operatorname{Gal}\left(K^{\mathrm{ab}} / K\right)$ on the moduli point $[X, \Phi]$, and thus gives a class-field-theoretic description of the action of $\operatorname{Gal}\left(K^{\mathrm{ab}} / K\right)$ on the 27 lines on $Y$.

\section{Acknowledgements}

Part of the material in Section 3 was developed in response to [7] and subsequent exchanges with its authors; it is a pleasure to thank J. Carlson and D. Toledo for their insights and encouragement. C. Hall and N. Katz kindly noted a mistake in an earlier work (see Remark 4.6), which is now corrected in Section 4.2. B. Gross pointed out that [13] contains a simple criterion for condition (3.4.1). Finally, this paper benefited from the referee's careful reading and concomitant suggestions. This work was partially supported by a grant from the Simons Foundation (204164).

\section{References}

[1] J.D. Achter, Arithmetic Torelli maps for cubic surfaces and threefolds (2012). To appear, Trans. AMS, 21 pages.

[2] J. D. Achter and R. Pries, The integral monodromy of hyperelliptic and trielliptic curves, Math. Ann. 338(1) (2007), 187-206.

[3] D. Allcock, J.A. Carlson and D. Toledo, The complex hyperbolic geometry of the moduli space of cubic surfaces, J. Algebr. Geom. 11(4) (2002), 659-724.

[4] Y. André, Mumford-Tate groups of mixed Hodge structures and the theorem of the fixed part, Composit. Math. 82(1) (1992), 1-24.

[5] C. Birkenhake and H. Lange, Complex abelian varieties, Grundlehren der Mathematischen Wissenschaften [Fundamental Principles of Mathematical Sciences] Vol. 302, Springer-Verlag, Berlin, 2nd. edition (2004), ISBN 3-540-20488-1.

[6] J.-F. Boutot and H. Carayol, Uniformisation p-adique des courbes de Shimura: les théorèmes de Čerednik et de Drinfel'd, Astérisque (196-197) (1991), 7, 45-158 (1992). Courbes modulaires et courbes de Shimura (Orsay, 1987/1988).

[7] J.A. Carlson and D. Toledo, Cubic surfaces with special periods, Proc. Amer. Math. Soc. 141(6) (2013), 1947-1962.

[8] A. Collino, The fundamental group of the Fano surface. I, II, in Algebraic threefolds (Varenna, 1981), Lecture Notes in Math. 947, 209-218, 219-220, Springer, Berlin (1982). 
[9] P. Deligne, Variétés de Shimura: interprétation modulaire, et techniques de construction de modèles canoniques, in Automorphic forms, representations and $L$-functions (Proc. Symp. Pure Mathematics, Oregon State University, Corvallis, Ore., 1977), Part 2, Proc. Symp. Pure Mathematics, XXXIII, 247-289, Amer. Math. Soc., Providence, R.I. (1979).

[10] — La conjecture de Weil. II, Inst. Hautes Études Sci. Publ. Math. (52) (1980), 137-252.

[11] I. Dolgachev, B. van Geemen and S. Kondō, A complex ball uniformization of the moduli space of cubic surfaces via periods of K3 surfaces, J. Reine Angew. Math. 588 (2005) 99-148.

[12] E. Z. Goren, Lectures on Hilbert modular varieties and modular forms, Vol. 14 of CRM Monograph Series, American Mathematical Society, Providence, RI (2002), ISBN 0-8218-1995-X. With the assistance of Marc-Hubert Nicole.

[13] B.H. Gross, Some remarks on signs in functional equations, Ramanujan J. 7(1-3) (2003), 91-93. Rankin memorial issues.

[14] A.J. Hahn and O.T. O'Meara, The classical groups and K-theory, Grundlehren der Mathematischen Wissenschaften [Fundamental Principles of Mathematical Sciences], 291, Springer-Verlag, Berlin (1989), ISBN 3-540-17758-2. With a foreword by J. Dieudonné.

[15] C. Hall, Big symplectic or orthogonal monodromy modulo l, Duke Math. J. 141(1) (2008), 179-203.

[16] J. Harris, Galois groups of enumerative problems, Duke Math. J. 46(4) (1979), 685-724.

[17] C. Jordan, Traité des substitutions et des équations algebriques, Gauthier-Villars, Paris (1870).

[18] N.M. Katz, Affine cohomological transforms, perversity, and monodromy, J. Amer. Math. Soc. 6(1) (1993), 149-222.

[19] R.E. Kottwitz, Points on some Shimura varieties over finite fields, J. Amer. Math. Soc. 5(2) (1992), 373-444.

[20] K.-W. Lan, Arithmetic compactifications of PEL-type Shimura varieties, number 36 in London Math. Soc. Monographs, Princeton University Press, Princeton, NJ (2013).

[21] K. Matsumoto and T. Terasoma, Theta constants associated to cubic threefolds, J. Algebraic Geom. 12(4) (2003), 741-775.

[22] J.S. Milne, Introduction to Shimura varieties, in Harmonic analysis, the trace formula, and Shimura varieties, Vol. 4 of Clay Math. Proc., 265-378, Amer. Math. Soc., Providence, RI (2005).

[23] — Class Field Theory (v4.01) (2011). Available at www.jmilne.org/math/.

[24] B. Moonen and Y.G. Zarhin, Hodge classes and Tate classes on simple abelian fourfolds, Duke Math. J. 77(3) (1995), 553-581.

[25] — Hodge classes on abelian varieties of low dimension, Math. Ann. 315(4) (1999), 711733.

[26] D. Mumford, Abelian varieties, Tata Institute of Fundamental Research Studies in Mathematics, No. 5, Published for the Tata Institute of Fundamental Research, Bombay (1970).

[27] J.-P. Serre, Topics in Galois theory, Vol. 1 of Research Notes in Mathematics, A K Peters Ltd., Wellesley, MA, second edition (2008), ISBN 978-1-56881-412-4. With notes by Henri Darmon.

[28] G. Shimura, On analytic families of polarized abelian varieties and automorphic functions, Ann. Math. (2) 78 (1963), 149-192.

[29] S.G. Tankeev, Algebraic cycles on abelian varieties. II, Izv. Akad. Nauk SSSR Ser. Mat. 43(2) (1979), 418-429.

[30] T. Terasoma, Complete intersections with middle Picard number 1 defined over $\mathbf{Q}$, Math. Z. 189(2) (1985), 289-296.

[31] J. Wilson, Degrees of polarizations on an abelian surface with real multiplication, Bull. Lond. Math. Soc. 33(3) (2001), 257-264.

Department of Mathematics, Colorado State University, Fort Collins, CO 80523, USA, http://www.math.colostate.edu/ achter

E-mail address: j.achter@colostate.edu 